\title{
Sickle cell disease in african immigrant children: A scoping review
}

\author{
Ziad Zahoui ${ }^{1}$,Higinio Fernandez Sánchez ${ }^{1}$, Michael Kariwo ${ }^{1}$, Bukola Salami ${ }^{1}$
}

${ }^{1}$ Department of Nursing, University of Alberta

\begin{abstract}
Despite the research on African-America parents, children and families who suffer from sickle cell disease (SCD), less is known about African immigrant children who migrated outside of Africa. Therefore, the purpose of this scoping review was to assess the extent, range, and nature of the existing index of research on African immigrant children with SCD living outside of Africa, to map out research activity and to identify gaps in the existing literature. This review followed the scoping review methodology of Arksey \& O'Malley. A comprehensive search in ten electronic databases was conducted. The search strategy combined the keywords "sickle cell" and "African immigrant children". Inclusion criteria focused on research published between 2000 and 2019, reporting on the health of African immigrant children aged 0 to 18 years. Systematic \& literature review reviews, conferences, case studies, viewpoint articles, \& epidemiology studies were excluded. Also, studies in which findings were based on mixed populations containing less than $80 \%$ African immigrant children, were excluded from the review.
\end{abstract}

This review was guided by the five-step approach to scoping reviews of Arksey \& O'Malley. Articles were included if they focused on African immigrant children living abroad. Two independent reviewers screened and selected articles. We analyzed and synthesized data using thematic analysis for qualitative data. The search yielded 6,602 records. After removing duplicates and titles, and abstracts were screened, 1,675 articles were included for full-text screening; 6 met inclusion criteria. Chain searching generated 10 articles, 1 met the inclusion criteria. Seven articles were included for analysis. The studies were all quantitative in design and none mentioned a theoretical framework. Even though all the studies were quantitative the usage of clinical assessment of the only means of attaining data, and the usage of insufficient sample size made the studies found, inconclusive in making generalizations. There are significant research gaps regarding African immigrant children with SCD living outside of Africa. A major limitation of the studies use is they were all conducted in Italy even though there are many other countries known to host immigrants from the African continent. Furthermore, most of the studies only state the disadvantages this population faces and only two studies took the initiative in addressing these issues. Overall, this review underlines the need for future research on the impact of migration on the health outcomes of African immigrant children with SCD living outside of Africa.

Key words:

Sickle cell, sickle cell disease, scoping review, African Immigrant children, Africa immigrant, migration, SCD

Cite as: Zahoui Z., Fernandez-Sánchez H., Kariwo M., Salami B. 2019. Sickle cell disease in African immigrant children: A scoping review. Alberta Academic Review, Vol 2 (2) 87-88, WISEST Special Issue (non peer-reviewed), DOI 10.29173/aar79. 


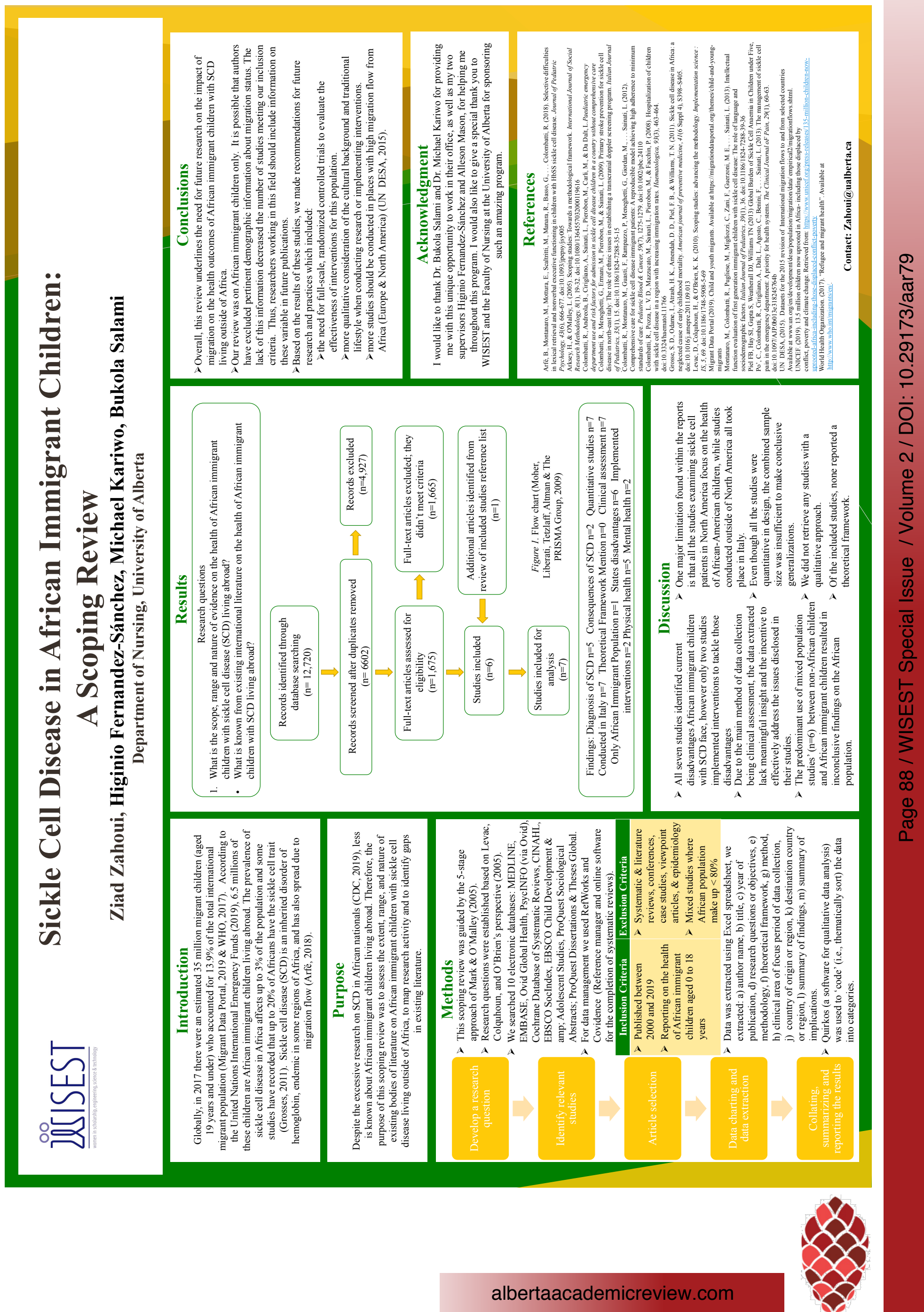

